\section{IN BRIEF}

\section{PROSTATE CANCER}

\section{Tissue penetration to optimize PSMA ADCs}

Systemic adverse effects of chemotherapy can be reduced by improving concentration of the agents at their site of action, a characteristic of antibody-drug conjugates (ADCs). Prostate-specific membrane antigen (PSMA)-binding singledomain ADCs were studied to determine the effect of tissue penetration on efficacy in vitro and in vivo. Interestingly, ADCs that demonstrated low potency in vitro had higher in vivo efficacy than high-potency ADCs. Smaller size of $\mathrm{ADC}$ and slower internalization rate improved tissue penetration and increased efficacy in vivo. Addition of an albumin-binding domain was also beneficial, as those lacking this domain were rapidly cleared, reducing cellular uptake. Thus, maximizing the number of cells that receive a lethal dose correlates with efficacy more strongly than total tumour uptake or in vitro potency. Modelling and protein engineering could be used to custom design ADCs for controlling these variables and maximizing outcomes.

ORIGINAL ARTICLE Nessler, I. et al. Increased tumor penetration of single-domain antibody drug conjugates improves in vivo efficacy in prostate cancer models. Cancer Res. https://doi.org/10.1158/0008-5472.CAN-19-2295 (2020)

\section{BLADDER CANCER}

\section{Genomic profiling suggests possible immunotherapy response in rare subtypes}

Therapy options are limited for patients with rare histologies of bladder cancer, such as adenocarcinoma of the bladder (ACB) and squamous cell carcinoma (SCC). Comprehensive genomic profiling was carried out on tumour samples from patients with ACB $(n=143)$, urothelial carcinoma (UC; $n=2,142)$ and SCC $(n=83)$, all of whom went on to develop advanced disease. Median tumour mutational burden (TMB) was significantly higher in UC and SCC than in ACB $(P<0.001)$. PD-L1 expression frequency in both tumour cells and tumour-infiltrating lymphocytes was higher in UC and SCC (30\%) than in ACB (18\%). SCC had the highest frequency of CD274 amplification. Thus, SCC has genomic features that suggest it might respond to immunotherapy in a similar way to UC, whereas ACB might be amenable to different targeted therapies.

ORIGINAL ARTICLE Necchi, A. et al. Comprehensive assessment of immuno-oncology biomarkers in adenocarcinoma, urothelial carcinoma, and squamous-cell carcinoma of the bladder. Eur. Urol. https://doi.org/10.1016/j.eururo.2020.01.003 (2020) RELATED ARTICLE Moschini, M. et al. Characteristics and clinical significance of histological variants of bladder cancer. Nat. Rev. Urol. 14, 651-668 (2017)

\section{PROSTATE CANCER}

\section{$B R C A 2$ and $R B 1$ loss responds to PARP inhibitors}

Molecular mechanisms and genomic consequences of BRCA2 and RB1 co-loss were prospectively investigated in prostate cancer cell lines. CRISPR-Cas9 and RNA interference (RNAi) methods were used to eliminate these genes in LNCaP and LAPC4 cells. Loss of BRCA2 led to a castrationresistant phenotype; co-loss of both genes induced epithelialto-mesenchymal transition (EMT). However, PARP inhibitors attenuated cell growth in human metastatic castrationresistant prostate cancer (CRPC) organoids and CRPC cells that had single-copy loss of both genes. Thus, identification of patients with co-loss of RB1 and BRCA2 could enable early use of PARP inhibition and improve outcomes.

ORIGINAL ARTICLE Chakraborty, G. et al. Significance of BRCA2 and RB1 co-loss in aggressive prostate cancer progression. Clin. Cancer Res. https://doi.org/10.1158/ 1078-0432.CCR-19-1570 (2020)

RELATED ARTICLE Taylor, R. A. et al. The influence of BRCA2 mutation on localized prostate cancer. Nat. Rev. Urol. 16, 281-290 (2019)

\title{
Mapping the male urogenital system
}

The male urogenital system has been comprehensively mapped on to the sensory cortex in the first study of its kind. These data can be used to improve our understanding of the sexual response in men.

Investigators used functional MRI to map primary somatosensory cortical responses to self-stimulation of the penis shaft, glans, testicles, scrotum, rectum, urethra, prostate, perineum and nipple. They also compared the neural responses to erotic and nonerotic touch of the penile shaft.

The study included 21 men with no urogenital or sexual health issues. These men were trained to rhythmically squeeze their penile glans, penile midshaft, scrotum, left testicle, right testicle and left nipple to achieve nonerotic touch. They were also asked to stimulate their penile shaft in a way they perceived as erotic, as erotic touch is subjective. A custom-made rod was used to stimulate the perineum, rectum and prostate; urethral stimulation was achieved using a thin intermittent urinary catheter. Participants were able to leave out any procedures they did not wish to perform and not all men completed each procedure. A post-scan questionnaire was administered to ascertain the participants' perception of each procedure.

The results showed that the response to mild stimulation of the penile glans or shaft was superficial in the paracentral lobule, whereas the location of the response to forceful squeezing was deep in the paracentral lobule. Direct stimulation of the urethra using a catheter caused a response that was distinct from forceful penile stimulation. Stimulation of the corpus cavernosum also seemed to cause a unique response in the deepest portion of the paracentral lobule.
The dorsomedial region of the paracentral lobule near the cortical surface was activated by mild rectal self-stimulation, whereas activation of a more posterior region of the dorsomedial paracentral region was evident when the anterior wall of the rectum was stimulated.

Activation of the posterior dorsomedial region of the paracentral lobule was observed after subtraction of the response to rectal stimulation from combined rectal-prostate self-stimulation, indicating that the rectum has a different homuncular projection region from the prostate.

The paracentral lobule near the cortical surface was activated on self-stimulation of the perineum and rectum. A region deep in the midline of the paracentral lobule was activated on stimulation of the scrotum or either testicle. The deep medial paracentral lobule region was activated on nipple stimulation, which partially overlapped with the region activated by penile or testicular stimulation.

The response pattern of intentional erotic self-stimulation of the penile shaft was markedly different from the response to nonerotic self-stimulation. Further analysis suggested that different physical touch does not account for differences between erotic and nonerotic stimulation.

The observations of the neural response to urogenital self-stimulation in this study increase our understanding of the sexual response in men and could help identify targets for treating urogenital and sexual arousal disorders.

Louise Stone

ORIGINAL ARTICLE Allen, K. et al. Male urogenital system mapped onto the sensory cortex: functional magnetic resonance imaging evidence.J. Sex. Med. https://doi.org/10.1016/ j.jsxm.2019.12.007 (2020) 